\title{
Temporal Epilepsy Causing Recurrent Abdominal Pain in Adults
}

\author{
Hassan Al-Hail1,2*, Stacy Schantz Wilkins1, Boulenouar Mesroua, ${ }^{1,2}$, Gayane Melikyan 1,2, \\ Nabil Azar1, Naim Haddad1,2, Basim Uthman"1,2, Maria Siddiqi1, Ameer Jan1, Rana Babur1, \\ Abdulraheem Alrabi' ${ }^{1}$, Dirk Deleu ${ }^{1,2}$, Gonzalo Alarcón ${ }^{1,2}$ \\ ${ }^{1}$ Neurology Section (Medicine Department) and Academic Health System, Hamad Medical Corporation, Doha, Qatar \\ ${ }^{2}$ Weill Cornell Medical College in Qatar, Education City, Qatar \\ Email: *halhail1@hamad.qa
}

How to cite this paper: Al-Hail, H., Wilkins, S.S., Mesroua, B., Melikyan, G., Azar, N., Haddad, N., Uthman, B., Siddiqi, M., Jan, A., Babur, R., Alrabi, A., Deleu, D. and Alarcón, G. (2018) Temporal Epilepsy Causing Recurrent Abdominal Pain in Adults. World Journal of Neuroscience, 8, 270-276.

https://doi.org/10.4236/wjns.2018.82021

Received: January 24, 2018

Accepted: May 8, 2018

Published: May 11, 2018

Copyright $\odot 2018$ by authors and Scientific Research Publishing Inc. This work is licensed under the Creative Commons Attribution International License (CC BY 4.0).

http://creativecommons.org/licenses/by/4.0/

\begin{abstract}
Abdominal epilepsy is often unrecognised as an epilepsy condition, particularly in adults. We present a rare adult patient who suffers daily episodes of abdominal pinching pain described lasting for 30 seconds, often evolving to loss of consciousness. Scalp EEG-video monitoring showed interictal left temporal slowing and frequent left temporal epileptiform discharges. Three stereotypical complex partial seizures consistent with her habitual attacks were recorded. Two seizures showed a left temporal onset and whereas the third one appeared to start on the right temporal lobe. In patients with paroxysms of abdominal pain, nausea or vomiting, abdominal epilepsy should be considered after exclusion of the most common aetiologies for gastrointestinal conditions.
\end{abstract}

\section{Keywords}

Focal Epilepsy, Abdominal Epilepsy, Temporal Lobe Epilepsy, Ictal

\section{Introduction}

Abdominal epilepsy is an uncommon syndrome in which paroxysmal symptoms resembling abdominal pathology result from seizure activity [1] [2]. Although abdominal sensations are common manifestations of seizures, symptoms resembling gastrointestinal conditions (such as abdominal pain, vomiting or diarrhea) are rare ictal symptoms, particularly in adults. Ictal pain is an uncommon ictal symptom, seen in as few as 2 per 1000 patients [3], and ictal abdominal pain is seen in only $33 \%$ of patients with ictal pain [4]. The syndrome of abdominal epilepsy is characterised by: 1) Otherwise unexplained, paroxysmal gastrointestinal complaints, mainly pain and vomiting; 2) Symptoms arise from a central nerv- 
ous system disturbance; 3) Abnormal EEG with findings specific for a seizure disorder; and 4) Improvement with anticonvulsant medication [5] [6]. A review of the history of this syndrome yielded 36 cases reported in the English literature in the previous 34 years [5].

The characteristic seizures in abdominal epilepsy consist of paroxysmal episodes of abdominal pain or diverse abdominal complaints associated with EEG abnormalities and usually favourable response to the introduction of anticonvulsants [1].

In the present report, we discuss one adult patient with recurrent abdominal pain, with associated symptoms suggestive of left temporal epilepsy, definite EEG and video-EEG abnormalities and a response to anticonvulsant drugs. The patient is a rare example of isolated abdominal pain with speech arrest as ictal manifestations. Patient's informed consent for publication was obtained.

\section{Clinical Report of the Case}

The patient is a 26 year-old, Arabic speaking, right handed female. Her birth and initial development were normal. She suffered febrile convulsions at 9 months. She did well at school until grade 6, after which her school performance deteriorated. At age 10, she suffered several complex partial seizures and later started to suffer episodes of head turning to the left and then right, with right arm tonic-clonic convulsions followed by generalization, episodes that later subsided. At present, she suffers the following episodes:

1) Episodes of aggressive behaviour associate with visual perceptions consisting of seeing a black colour and/or figures of ghosts.

2) Abdominal pain described as pinching, lasting for approximately 30 seconds, often associated with inability to speak and followed by post-ictal confusion with excessive eating for which she has no recall. This is the most frequent seizure type, occurring almost every day.

3) Episodes of confusion and disorientation.

Since investigations for gastrointestinal conditions were normal, epilepsy was suspected. The frequency of episodes reduced by $30 \%$ on lamotrigine ( 400 $\mathrm{mg} /$ day) and lacosamide (200 mg/day). A brain MRI showed left mesial temporal sclerosis (Figure 1). An EEG showed mild diffuse slowing of the background activity and left anterior temporal epileptiform discharges.

At present she does not work and spends her time at home with her family. She enjoys reading. Her sleep wake cycle is reportedly reversed, sleeping during the day and remaining awake until late at night, partly due to fear of "ghosts" and the dark. Neuropsychological assessment was not localizing and the patient demonstrated slow processing, with low average performance in both visual and verbal memory, low average visuospatial integration skills, and mild symptoms of depression.

The patient was referred to the epilepsy monitoring unit for continuous video-EEG recording and monitored for 4 days. The interictal EEG showed inter- 
mittent left temporal slowing and frequent left anterior and posterior epileptiform discharges during sleep and wakefulness, in addition to rare independent right sided discharges (Figure 2a). Three complex partial seizures were recorded which were of similar semiology and consistent with her habitual attacks. (Figure 2b) During the attacks, she would refer an aura of abdominal pain, lose awareness and show a distressed look, engage in repetitive moaning and body rocking, followed by motionless staring lasting for approximately one minute. The episodes were followed by brief confusion and relatively quick recovery. The concomitant ictal EEG recordings demonstrated that the attacks were epileptic seizures. Two of the seizures showed a left temporal onset and evolution, whereas the third one appeared to start on right temporal region, evolving later to the left side. The patient also experienced one event with subjective feelings of fear, derealisation and abdominal pain without alteration of language or awareness. There were no concomitant EEG changes during this event which likely represented a simple partial.

\section{Discussion}

In the present case, we found that the most common ictal symptoms were paroxysmal abdominal pain, speech arrest and visual aura. The key to diagnosis was provided by symptoms suggestive of functional abdominal pain in association with definite EEG and video-EEG abnormalities. We report here ictal recordings in abdominal epilepsy (Figure 2(b)), which to our knowledge has only been reported once before [7].

Abdominal epilepsy is well documented among children, but is infrequently recognised in adults [8]. The clinical presentation of abdominal epilepsy is characterised by paroxysmal episodes with both gastrointestinal and central nervous system (CNS) manifestations [9] [10]. The gastrointestinal manifestations include all or a combination of the following: recurrent abdominal pain, nausea, vomiting, bloating or diarrhoea. A wide diversity in CNS manifestations has also been reported, which include confusion, fatigue, headache, dizziness and syncope. Although abdominal symptoms may be similar to those of irritable bowel syndrome, abdominal epilepsy may be distinguished from the latter by the presence of altered consciousness during some of the episodes, a tendency towards tiredness after an episode, and presence of interictal or ictal EEG abnormalities.

Previous reports suggest that the most common interictal EEG abnormalities in patients with abdominal epilepsy are bursts of sharp waves or spikes over one or both temporal lobes [1] [8]. A video-EEG is a standard non-invasive investigation which may be crucial for the differential diagnosis between gastrointestinal conditions and abdominal epilepsy, since patients with abdominal epilepsy often have ictal EEG abnormalities, particularly arising from temporal lobe structures, as was the case in our patient. Abdominal ictal symptoms such as retching, flatulence and urge to urinate are usually interpreted as insular symptoms arising from the non-dominant hemisphere. However the lateralising value 

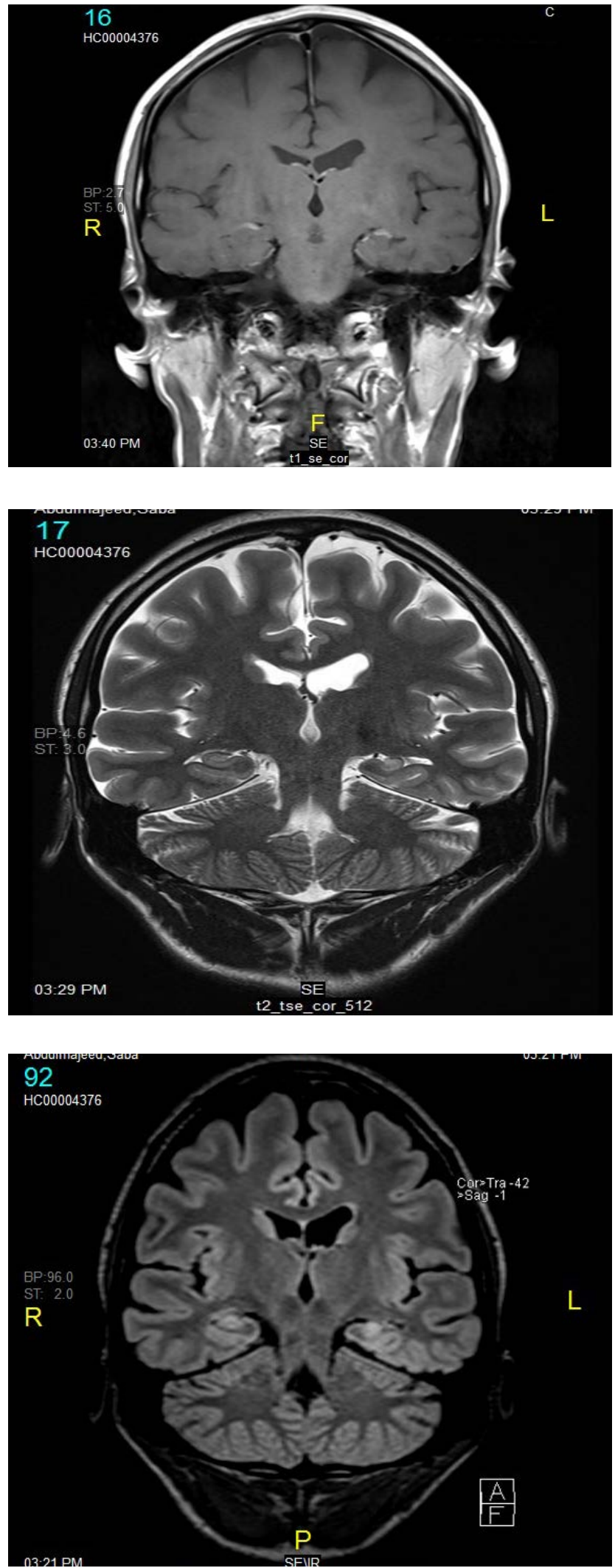

Figure 1. Magnetic resonance images. Coronal T1 and T2 weighted images and FLAIR image showing left mesial temporal sclerosis. Note the smaller (T2) and brighter (FLAIR) left hippocampus. 


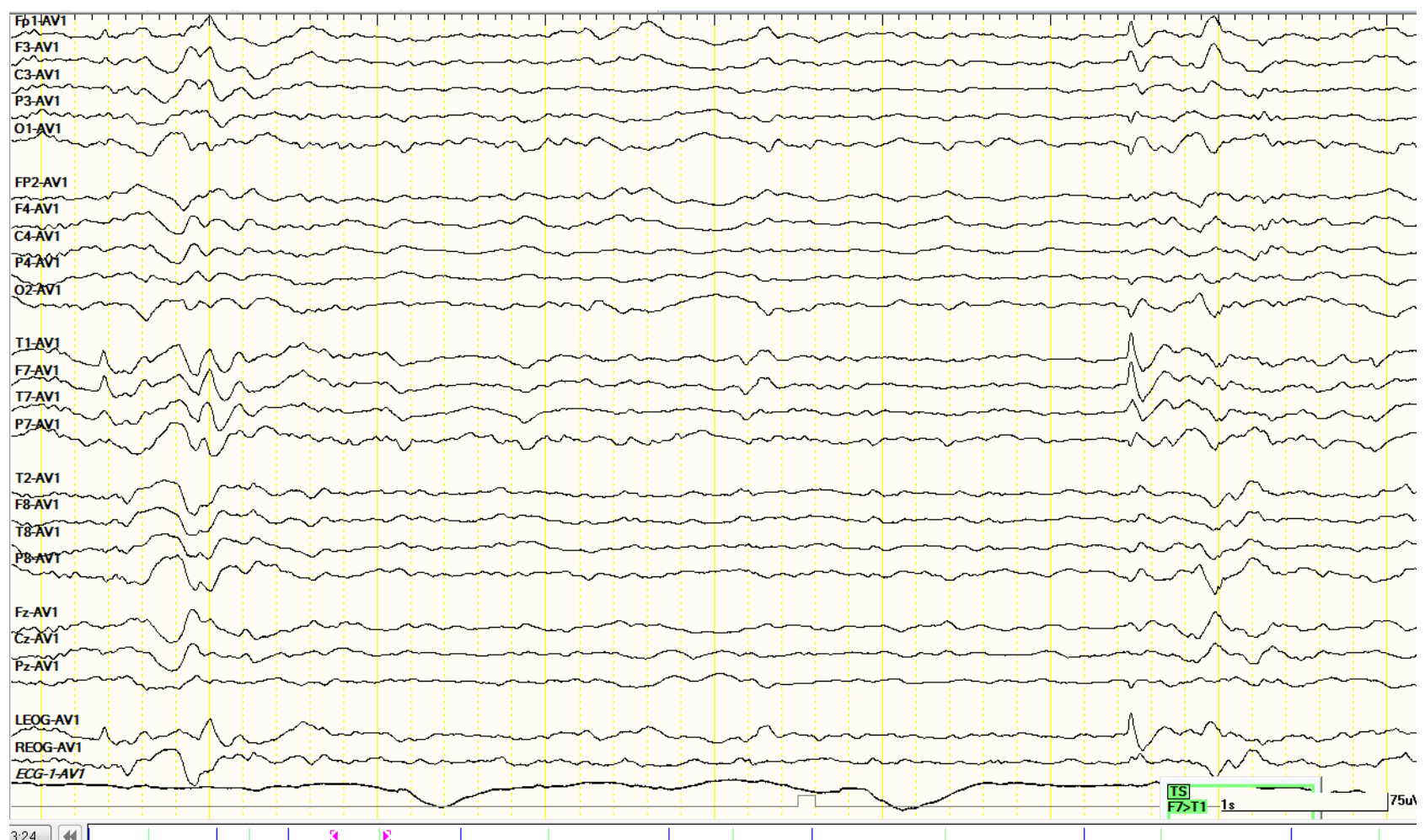

(a)

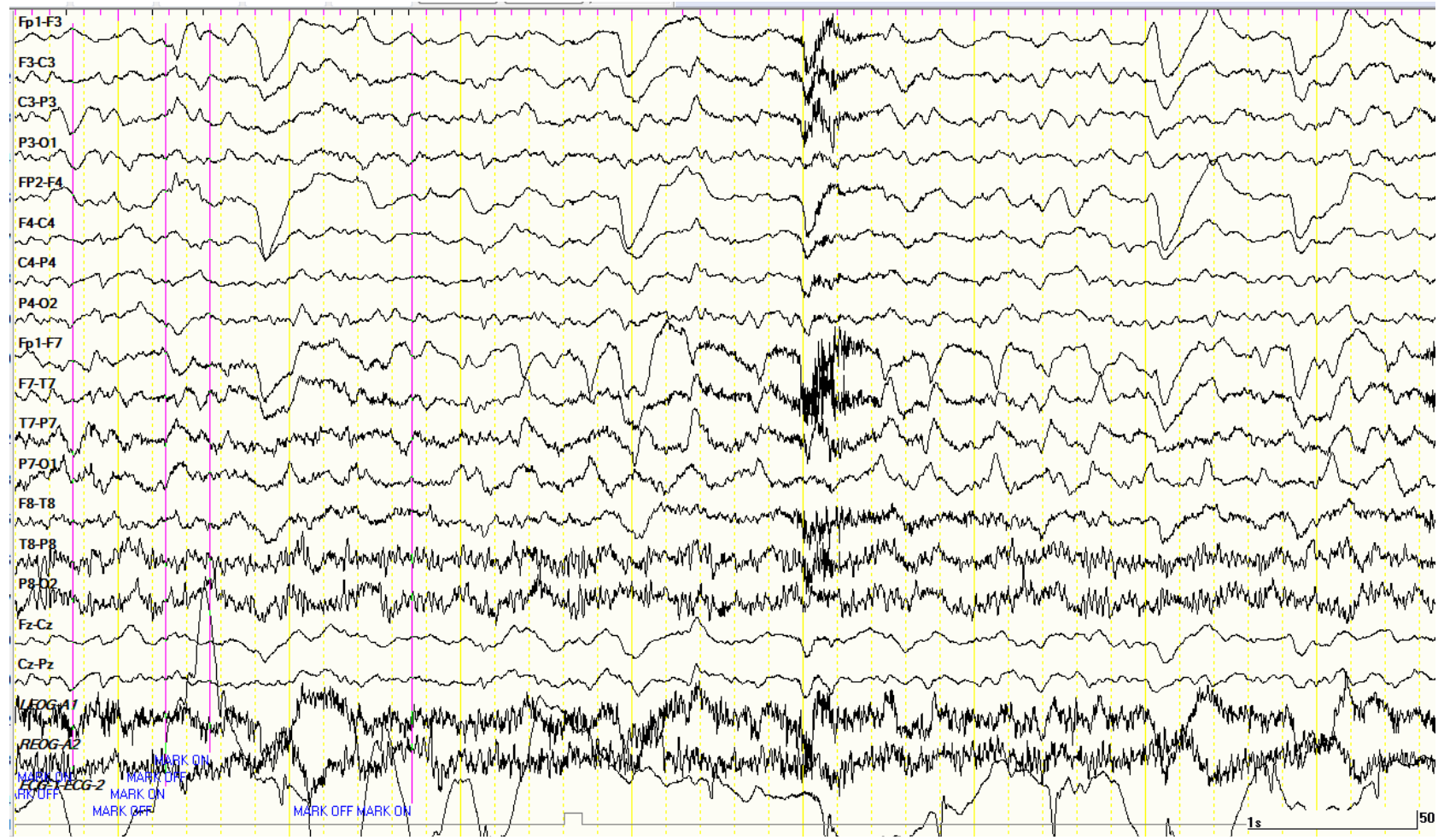

(b)

Figure 2. (a) EEG recording showing left anterior temporal interictal epileptiform discharges; (b) Ictal EEG recording showing left anterior temporal onset. 
of abdominal pain is less clear. Most series describing abdominal epilepsy do not report the laterality of brain abnormalities. Many patients show bitemporal independent discharges [5]. There is one case report of ictal diarrhea arising from the left hemisphere [10].

A sustained response to anticonvulsants has been accepted as one of the diagnostic criteria for abdominal epilepsy [8]. However, there are no recommendations on the choice of anticonvulsants. Our patient was improved on lamotrigine and lacosamide.

\section{Conclusion}

The present case demonstrates that diagnosis of abdominal epilepsy is particularly challenging: not only ictal symptoms can be vague and non-specific, but also some simple partial seizures may not show clear ictal EEG changes. In addition, the condition is rare and often not thought of, particularly in adults. We suggest that in patients with paroxysms of abdominal pain, nausea and vomiting with or without CNS manifestations, the possibility of abdominal epilepsy should be considered after exclusion of the most common aetiologies for gastrointestinal conditions. The investigation in these patients should proceed with an EEG and possibly ictal video-EEG. Treatment typically begins with anticonvulsant drugs. Lacosamide and lamotrigine appear to be effective anticonvulsants in our case.

\section{Clinical Message}

- The possibility of abdominal epilepsy should be considered after exclusion of the most common aetiologies for gastrointestinal conditions.

- Abdominal epilepsy may be distinguished from the bowel conditions by the presence of altered consciousness during some of the episodes, a tendency towards tiredness after an episode, and presence of interictal or ictal EEG abnormalities.

- We report here the first ictal recordings in abdominal epilepsy (Figure 2(b)).

- Abdominal ictal symptoms such as retching, flatulence and urge to urinate are usually interpreted as insular symptoms arising from the non-dominant hemisphere, but the possibility of temporal lobe epilepsy should also be considered.

- Lacosamide and lamotrigine can be effective anticonvulsants in abdominal epilepsy.

\section{References}

[1] Franzon, R.C., Lopes, C.F., Schmutzler, K.M., et al. (2002) Recurrent Abdominal Pain: When Should an Epileptic Seizure Be Suspected? Arq Neuropsiquiatr, 60, 628-630. https://doi.org/10.1590/S0004-282X2002000400021

[2] Ostwal, P. (2013) Abdominal Epilepsy: Is It So Common? Journal of Pediatric Neurosciences, 8, 253. https://doi.org/10.4103/1817-1745.123698 
[3] Kuloğlu Pazarc1, N., Bebek, N., Baykan, B., Gürses, C. and Gökyiğit, A. (2016) Reappraisal of Epileptic Pain as a Rare Symptom of Seizures. Epilepsy \& Behavior, 55, 101-107. https://doi.org/10.1016/j.yebeh.2015.12.015

[4] Bulacio, J., Klem, G. and Lüders, H.O. (2000) Painful Auras. In: Luders, H.O. and Noachtar, S., Eds., Epileptic Seizures. Pathophysiology and Clinical Semiology, Churchil Livingstone, New York.

[5] Zinkin, N.T. and Peppercorn, M.A. (2005) Abdominal Epilepsy. Best Practice \& Research Clinical Gastroenterology, 19, 263-274. https://doi.org/10.1016/j.bpg.2004.10.001

[6] Dutta, S.R., Hazarika, I. and Chakravarty, B.P. (2007) Abdominal Epilepsy, an Uncommon Cause of Recurrent Abdominal Pain: A Brief Report. Gut, 56, 439-441. https://doi.org/10.1136/gut.2006.094250

[7] Shihabuddin, B.S. and Harik, S.I. (2007) Episodic Nausea and Abdominal Sensations as Sole Manifestations of Simple Partial Seizures. Neurosciences (Riyadh), 12, 327-329.

[8] Murai, T., Tohyama, T. and Kinoshita, M. (2014) Recurrent Diarrhea as a Manifestation of Temporal Lobe Epilepsy. Epilepsy \& Behavior Case Reports, 19, 57-59. https://doi.org/10.1016/j.ebcr.2014.03.007

[9] Peppercorn, M.A. and Herzog, A.G. (1989) The Spectrum of Abdominal Epilepsy in Adults. American Journal of Gastroenterology, 84, 1294-1296.

[10] Zarling, E.J. (1984) Abdominal Epilepsy: An Unusual Cause of Recurrent Abdominal Pain. American Journal of Gastroenterology, 79, 687-688. 